\title{
The cortical projection upon the claustrum ${ }^{1}$
}

\author{
J. B. CARMAN, W. M. COWAN, AND T. P. S. POWELL \\ From the Department of Human Anatomy, Oxford
}

Comparatively little is known about the connexions of the claustrum; two recent reviews (Rae, 1954; Berke, 1960) have summarized the evidence available from studies of normal and Marchi-stained material, and have emphasized the inadequacy of our understanding of the basic organization of the connexions of the claustrum. In view of the paucity of knowledge it was considered worthwhile to place on record the following observations on the cortical afferents to the claustrum derived from a large series of rabbit brains in which a variety of cortical lesions had been placed, prepared by the Nauta and Glees methods. The material was originally collected for a study of

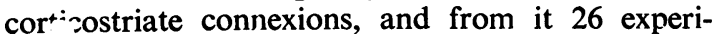
ments have been selected for the present investigation. Several of these brains have been described previously, and the details regarding the placement of the lesions and the histological preparation have been given in full elsewhere (Carman, Cowan, and Powell, 1963). In each case the site and extent of the cortical lesion has been carefully reconstructed and transferred to a standard diagram of the rabbit hemisphere. The distribution of the fibre degeneration in the claustrum is similarly shown on a standard series of drawings from the projected outlines of a normal brain. To determine the basic organization of the projection of the cortex upon the claustrum it is convenient to divide the hemisphere into five topographic regions, and in the following account only one or two examples from each of these regions will be described.

Experiment R48 is typical of several similar cases with lesions on the dorso-lateral aspect of the hemisphere and serves to illustrate many of the characteristic features of the cortico-claustral projection. The site and extent of the lesion are shown in Fig. 1; the area of cortical damage is roughly oval in outline and occupies the central part of the posterior half of the hemisphere immediately lateral to the 'cingular sulcus'. Throughout the greater part of its extent it has penetrated deeply into the white matter but has not involved the hippocampus. In continuity with the lesion severe

${ }^{1}$ This work was supported by a grant from the Medical Research Council. fibre degeneration can be traced downwards and laterally in the corona radiata to the dorsal end of the claustrum. Here it separates into two components, the medial of which passes into the external capsule, and the lateral into the subcortical white matter on the outer aspect of the claustrum. From both components degenerating fibres can be seen passing into the claustrum; those from the external capsule are directed obliquely downwards and laterally through the upper third of the claustrum, while those from the subcortical white matter extend horizontally inwards at the level of its middle third. Both groups of fibres terminate as a dense pericellular plexus amongst the cells of the middle third of the dorso-ventral extent of the claustrum. The antero-posterior distribution of the degeneration in the claustrum correspond 8 closely to that in the caudate nucleus, extending $P$ from a level just behind the middle of the head of $\mathbb{O}$ the caudate back almost to the caudal end of it body. The ventral third of the claustrum is free o⿺ degeneration throughout its extent, and there is nes difficulty in distinguishing the preterminal degenera? tion seen in its middle third from the degenerating fibres of passage found in the upper third. Similarly, the pattern of degeneration in the claustrum is quite different from that seen in the overlying cortex where the degenerating fibre fragments have a characteristically radial distribution, paralleling the essentially columnar arrangement of the cells.

Only one other experiment, R96, with a lesion in the intermediate part of the dorsal aspect of the hemisphere, will be described because it demonstrates that a more anteriorly placed lesion results in degeneration in more rostral parts of the claustrum and that in the Glees preparations which are available for this brain the area of the terminal degeneration coincides with that showing preterminal degeneration in the Nauta sections. As may be seen from Fig. 2, the lesion lies in the intermediate part of the dorsal surface but is more anterior than that in the previous experiment; however, the caudal part of the lesion overlaps that of R48, and the underlying white matter has been involved. In the claustrum the distribution of preterminal degeneration again parallels that seen in the striatum, extend- 

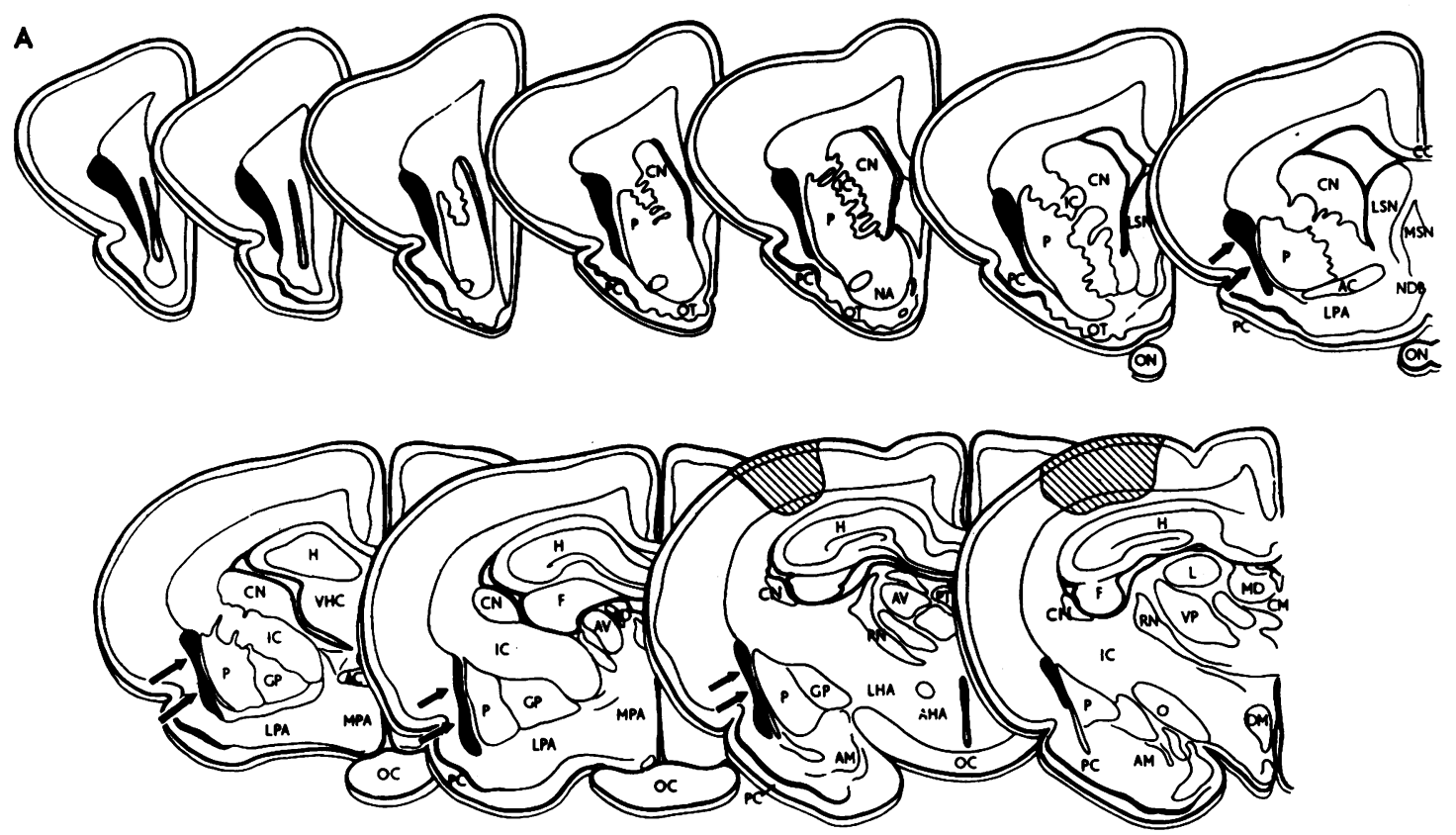

B
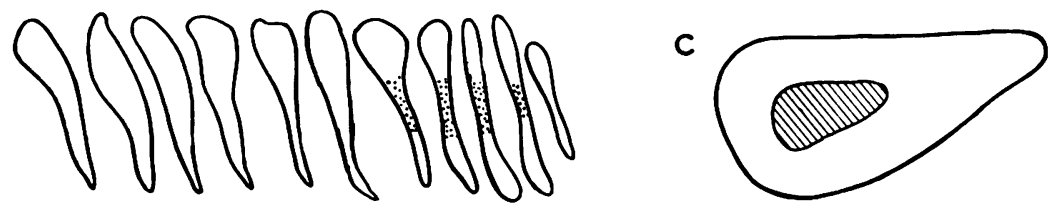

FIG. 1. The site of the lesion and the distribution of the degeneration in the claustrum in experiment $\mathrm{K48.}$ In $A$ the claustrum is shown in a regularly spaced series of coronal sections through the hemisphere, and the areas containing preterminal degeneration are indicated by arrows. In $B$ the distribution of degeneration within the enlarged outlines of the claustrum is shown by stippling, and $C$ is a reconstruction of the lesion as seen on the dorsal surface of the hemisphere. In this and the subsequent figures the lesion is shown by cross-hatching, and the degeneration in the claustrum either by stippling or by bold arrows.

AC Anterior commissure

AHA Anterior hypothalamic area

AM Amygdala

AV Anteroventral nucleus

CC Corpus callosum

CM Nucleus centralis medialis

CN Caudate nucleus

DM Dorsomedial nucleus of hypothalamus

F Fimbria

GP Globus pallidus

H Hippocampus

IC Internal capsule

$L \quad$ Lateral nucleus

LHA Lateral hypothalamic area

LPA Lateral preoptic area
LSN Lateral septal nucleus

$M D \quad$ Dorsomedial nucleus of thalamus

MPA Medial preoptic area

MSN Medial septal nucleus

$N A \quad$ Nucleus accumbens

$N D B$ Nucleus of the diagonal band

O Optic tract

OC Optic chiasma

ON Optic nerve

OT Olfactory tubercle

$P \quad$ Putamen

PC Pyriform cortex

$P T \quad$ Parataenial nucleus

RN Reticular nucleus

VHC Ventral hippocampal commissure 

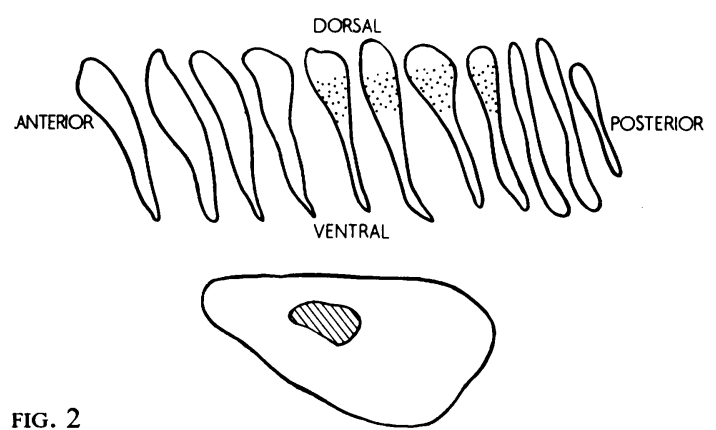

FIG. 2

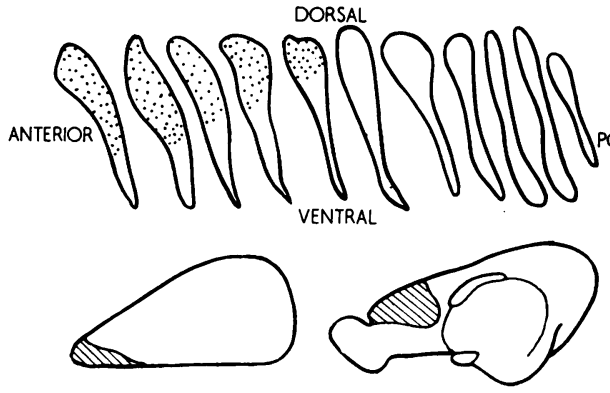

FIG. 3

FIG. 2. The position of the lesion and the extent of the degeneration in the claustrum in experiment R96.

FIG. 3. The position of the lesion as seen on the dorsal and medial surfaces of the hemisphere, and the distribution of the $\underset{\mathbb{D}}{\mathbb{D}}$ degeneration in the claustrum in experiment $R 82$.

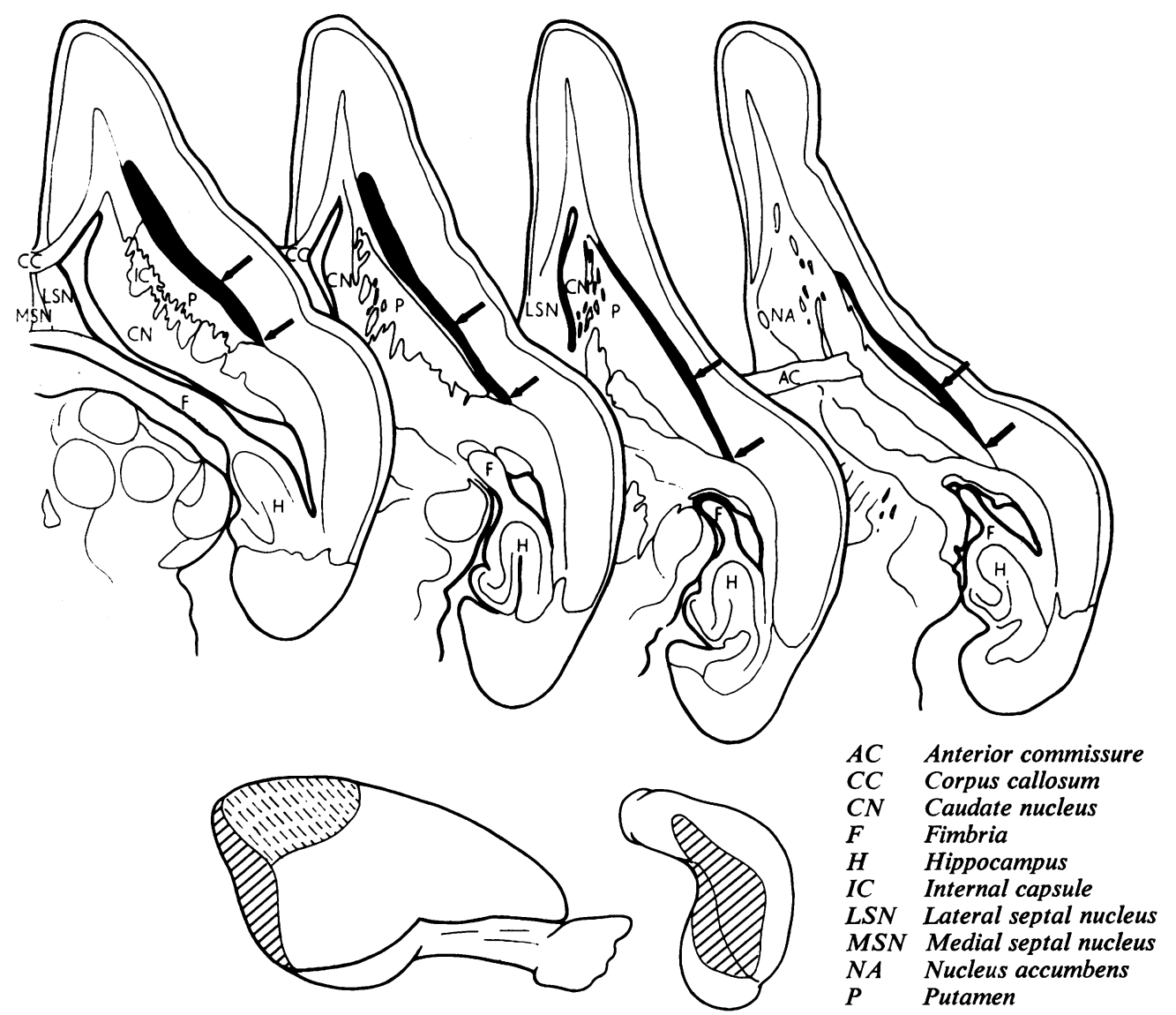

FIG. 4. The extent of the lesion as seen on the lateral and posterior aspects of the hemisphere (the area of broken hatching $N^{\circ}$ indicates the area of cortex undercut by the deep extension of the lesion) in experiment R107. The distribution of the degeneration in the claustrum is shown in the horizontal sections of the hemisphere. 
ing between the level of the anterior end of the septum and the interventricular foramen. In the sections stained by the Glees method numerous degenerating fibres are seen in the upper part of the claustrum, but further ventrally there are many typical swollen end-bulbs lying on the surface of the cells.

Experiment R82 is typical of those cases with lesions in the frontal region of the hemisphere. Here the cortex on the dorsal and medial surfaces has been destroyed from the frontal pole back almost to the level of the genu of the corpus callosum (Fig. 3). In the claustrum degeneration is seen in the dorsal half of its cross-sectional area from its rostral end as far caudally as the level of the anterior end of the septum. Once more this corresponds to the antero-posterior extent of the degeneration in the striatum, and in both the claustrum and the striatum the extent of the degeneration appears to be disproportionately large compared with the amount of the cortical damage.

To complete the evidence for an antero-posterior organization in the cortical connexions to the claustrum only one further experiment will be

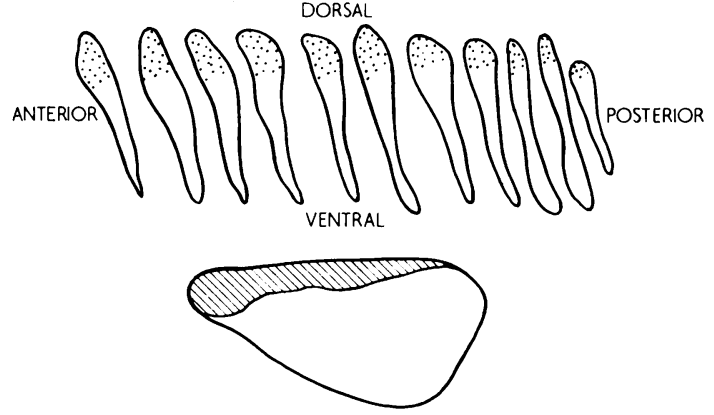

FIG. 5. The lesion and the resulting degeneration in the claustrum in $R R 2$.

described. In the brain of R107 the cortex along the posterior aspect of the hemisphere was removed, and a deep extension of the lesion has resulted in the interruption of fibres from a more extensive area on the postero-dorsal surface (Fig. 4). In the horizontal sections of this brain preterminal degeneration is found in the posterior third of the claustrum, and although this extends over most of its dorso-ventral
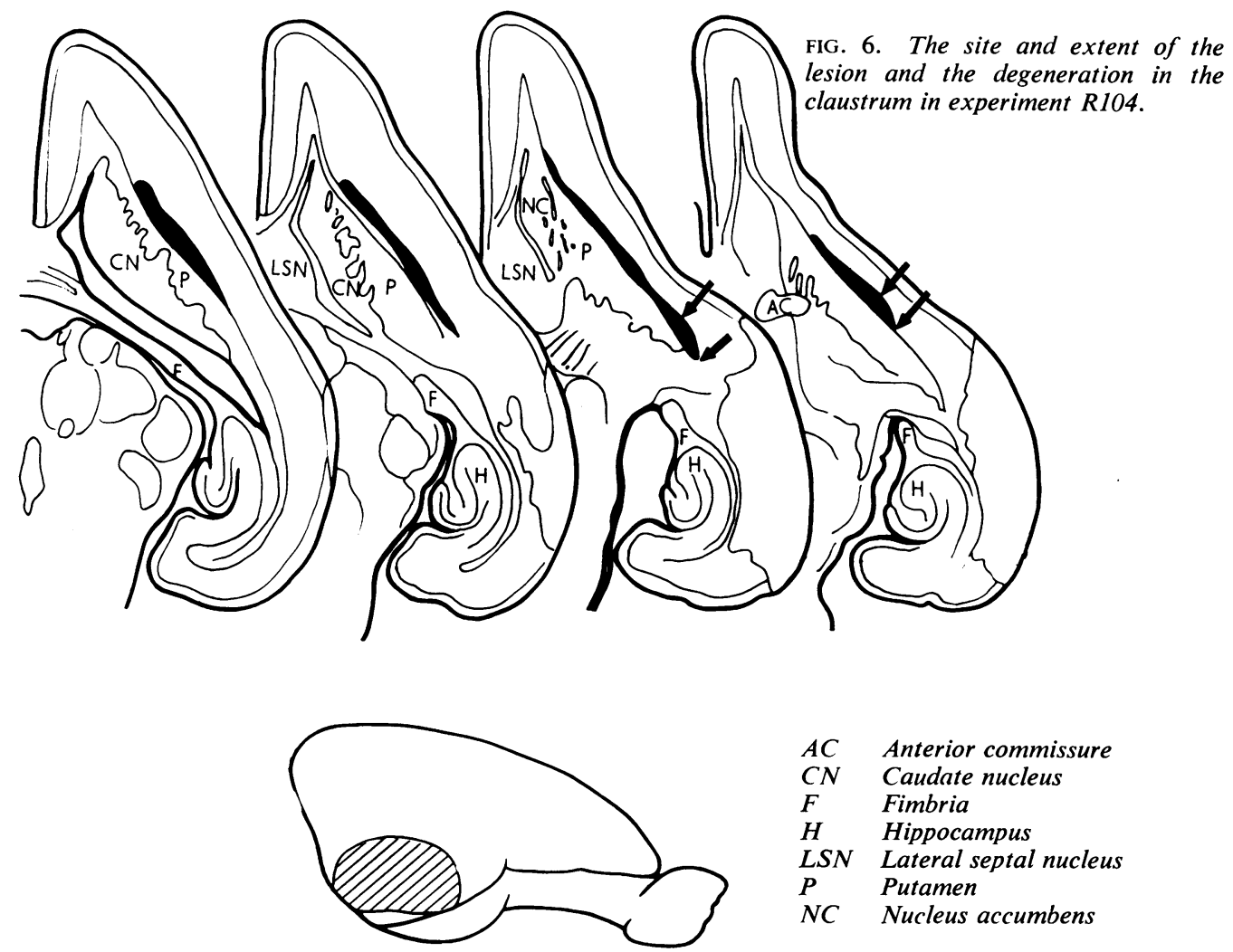


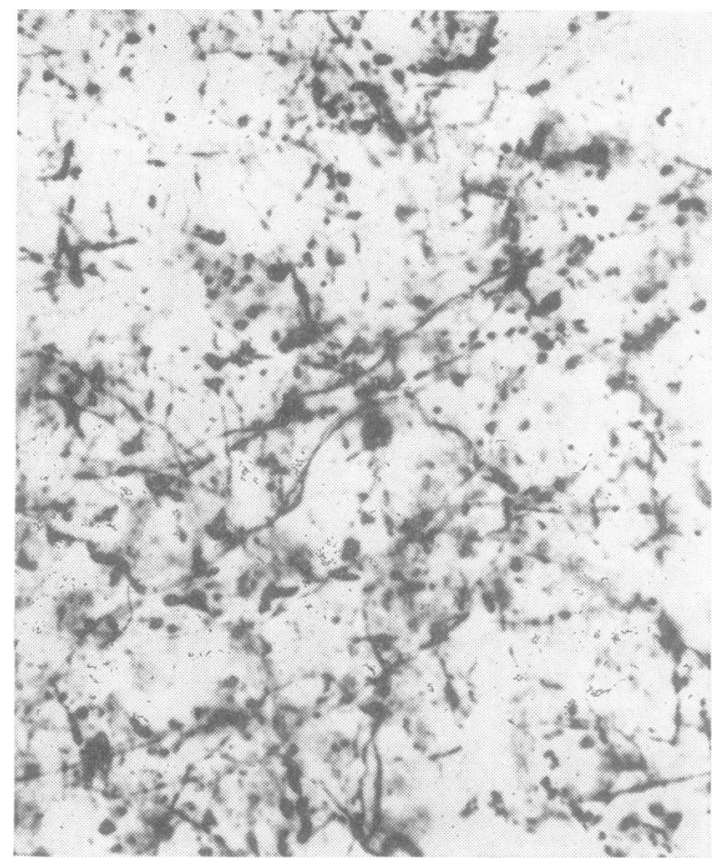

FIG. 7

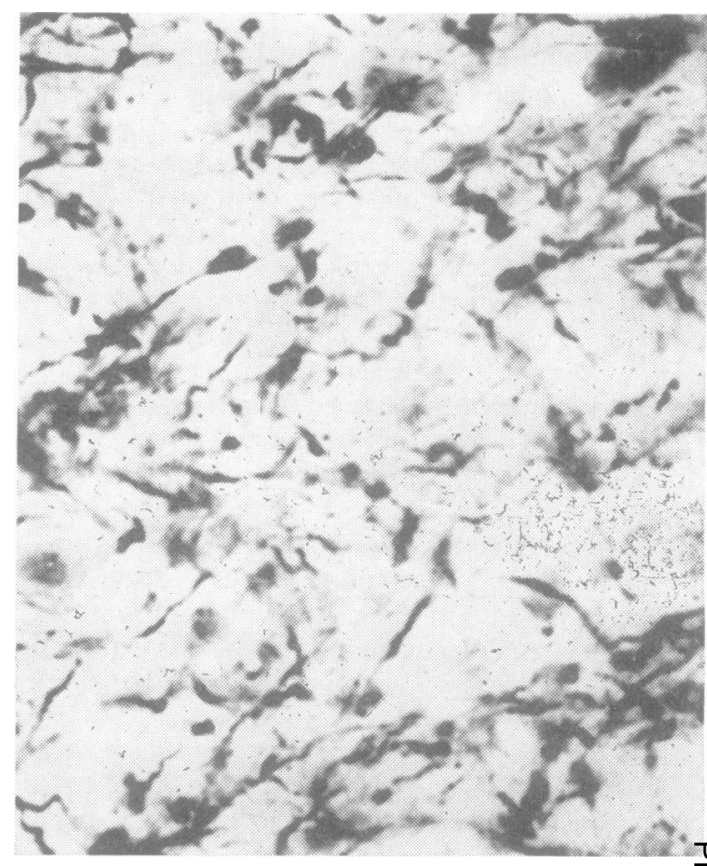

FIG. 8

FIG. 7. Photomicrograph to show preterminal degeneration in the central part of the claustrum in experiment R96 sevel
days after a lesion of the dorso-lateral aspect of the hemisphere. Nauta method. $\times 790$. FIG. 8. Photomicrograph to show degeneration of fibres and terminal boutons in the claustrum in the same experimen $\mathbb{D}$ Glees method. $\times 1,320$.

extent the density is greatest in the middle third. This experiment is of interest not only in showing that the posterior part of the hemisphere is related to the corresponding posterior part of the claustrum, but it is also the first case in which the degeneration has affected the entire dorso-ventral extent of the claustrum. This is probably due to the lateral extension of the lesion and suggests that there is a medio-lateral organization in the cortico-claustral projection. That this is so will be demonstrated by the findings in the next two brains.

In rabbit RR2 the cortical damage is in the form of a longitudinal strip along the dorso-medial margin of the hemisphere reaching from the frontal pole almost to its caudal end. The degeneration in the claustrum similarly extends over most of its antero-posterior extent, but it is confined throughout to its dorsal third; in the rostral part of the claustrum the pericellular preterminal degeneration occupies its entire width, but posteriorly only the medial margin adjacent to the external capsule is affected. Experiment R104 provides an excellent demonstration of the degree of localization that is present in the cortico-claustral projection as well as establishing that the lateral part of the cortex is con- nected with the ventral part of the claustrum. The. cortical lesion (Fig. 6) lies immediately above the $\mathscr{O}$ posterior part of the rhinal sulcus, its deep part extending forwards almost to the level of the posterior end of the claustrum. In the latter structure preterminal degeneration is only found in the most ventral sections, and on these, in turn, it is confined to the posterior quarter.

The question whether the degeneration seen in these experiments is that of fibres actually terminating in the claustrum need not be discussed as the appearance conforms in all respects with the generally accepted criteria for terminal degeneration. In addition, it is in agreement with recent incidental reports of degeneration within the claustrum following cortical lesions (Whitlock and Nauta, 1956; Black and Myers, 1962). It is of interest that the cortical projection upon the claustrum is so similar to that of the corticostriate projection (Carman, Cowan, and Powell, 1963). Thus in both cases it appears that in the rabbit, at least, all areas of the cerebral cortex send fibres to these two basal ganglia, and conversely that all parts of the claustrum and striatum receive afferents from the cortex. In both projections, also, there is a well-defined topo- 
graphic organization in that anterior and posterior parts of the cortex project to corresponding regions of the claustrum, and a line drawn along the cortex from the dorso-medial margin to the rhinal sulcus would be represented by a dorso-ventral line in the claustrum. A further similarity in the cortical projection to the claustrum and striatum is that in both cases the frontal cortex is related to a substantially larger volume of these basal ganglia than any other cortical region.

\section{REFERENCES}

Berke, J. J. (1960). J. comp. Neurol., 115, 297.

Black, P., and Myers, R. E. (1962). Anat. Rec., 142, 216.

Carman, J. B., Cowan, W. M., and Powell, T.P.S. (1963). Brain, 86, 525.

Rae, A. S. L. (1954). Confin. neurol. (Basel), 14, 211.

Whitlock, D. G., and Nauta, W. J. H. (1956). J. comp. Neurol.,\{106, 183. 